\title{
THE COGNITIVE NEUROSCIENCE OF OBJECT REPRESENTATION AND RECOGNITION
}

\section{Preface}

How do humans and nonhuman primates recognize objects? The difficulty in answering this question arises from not only the large number of objects we can recognize but also the wide range of situations in which objects appear. Consider how many faces you can recognize. Then consider how the retinal image of a specific face changes under different lighting conditions, under different distances between you and the person you're recognizing, and so forth. Yet, despite the large number of faces you can recognize and the physical conditions under which you can recognize these faces, recognition seems to occur quickly and easily. Researchers across many disciplines, including computer science, neuroscience, and psychology, are working toward an understanding of recognition mechanisms. I organized this special issue of Psychobiology to capture some of the recent developments in the understanding of object representation and recognition. The emerging field of cognitive neuroscience has provided an ideal environment for the study of object recognition through encouraging the use of varied methodologies to converge on the computational, cognitive, and neural mechanisms that allow shapes to be identified.

Because of the interdisciplinary nature of cognitive neuroscience, the individual papers appearing in this special issue rely on many different methodologies. Also, because the readership of Psychobiology is broad, most readers will not be specialists in the field of object recognition. For these two reasons, in the first article, titled "Visual Object Representation: An Introduction," I present an overview of some of the key findings from and issues in the cognitive neuroscience of object recognition. This introduction is organized around the computational, behavioral, and neural mechanisms of shape recognition, providing the reader with an introduction to results from different disciplines and how these results can be integrated with one another.

Several of the papers in the issue use neuroimaging techniques, techniques that have become increasingly popular for studying the neural mechanisms of object representation. In "Toward Direct Visualization of the Internal Shape Representation Space by fMRI," Edelman, Grill-Spector, Kushnir, and Malach integrate recent computational approaches to object representation with fMRI methods. By applying multidimensional scaling to their fMRI data, Edelman et al. show that blood flow patterns can represent the similarities among different objects: Blood flow patterns associated with two images of different cars are more similar than an image of a car and an image of an airplane. In the paper following Edelman et al., titled "Human Visual Object Recognition: What Have We Learned From Neuroimaging?" Aguirre and Farah present a meta-analysis of several neuroimaging studies. Like Edelman et al., Aguirre and Farah look to existing neuroimaging data to determine whether structural similarities across objects can be revealed through blood flow data. Through their analysis of 17 imaging studies, Aguirre and Farah find little evidence to suggest that localization of blood flow to a specific object category (e.g., words or faces) is consistent from one study to the next. These results raise important implications for the interpretation of neuroimaging studies, particularly when the interpretation is across different studies.

The next two papers in the issue represent applications of neuroimaging techniques. In "Neural Systems That Encode Categorical Versus Coordinate Spatial Relations: PET Investigations," Kosslyn, Thompson, Gitelman, and Alpert use positron emission tomography to study the neural mechanisms of different types of object representation. Kosslyn's previous work has identified "categorical" and "coordinate" representations. Categorical representations code for the category that describes the spatial relations between object parts, such as a person's head being located "above" the torso. Coordinate representations code for metric properties, such as the precise 
distance between an object's part and an origin, such as a person's nose (the part) being located $10.5 \mathrm{~cm}$ from the top of the head (the origin). Consistent with results from neurologically intact subjects and neuropsychological patients, Kosslyn et al. report that different brain regions are involved in categorical and coordinate representations. Next, in "Human Brain Potentials Related to Emotional Expression, Repetition, and Gender of Faces," Marinkovic and Halgren employ event related potentials (ERPs) to study the brain mechanisms of face processing. Specifically, Marinkovic and Halgren ask whether the mechanisms for processing facial identity are separate from the mechanisms for processing facial expression (emotional expression). Their results indicate that different ERP components are sensitive to identity and emotional manipulations, suggesting that face identity and face expression may be processed by different brain regions.

Although neuroimaging methods are currently en vogue in cognitive neuroscience, these methods do not represent the only technique for studying the neural bases of object processing. The final two papers in the issue demonstrate nicely the importance of other methodologies in studying object recognition. For example, Peterson, Gerhardstein, Mennemeier, and Rapcsak's paper, "Object-Centered Attentional Biases and Object Recognition Contributions to Scene Segmentation in Left- and Right-Hemisphere Patients," present data from neuropsychological patients that potentially constrain theories of visual processing. In their study, Peterson and colleagues investigated object-centered effects in neuropsychological patients with attentional deficits. The patients in this study exhibited a bias to pick regions in the contralesional visual field (i.e., the visual field opposite the lesioned hemisphere) as objects. These results indicate that attention may be allocated to objects, not to spatial locations. A further study by Peterson et al. demonstrates that the patients were more likely to pick familiar regions as object when the region depicted a familiar object; this result occurred even when the familiar region appeared in the unbiased visual field (i.e., the ipsilesional field). These results point to the complex interplay between object and spatial information in the visual system and may support the hypothesis that some object recognition occurs preattentively. Finally, in "Illumination Effects in Face Recognition," Braje, Kersten, Tarr, and Troje use tried-and-true behavioral methods with neurologically normal subjects to investigate face recognition processes. Interestingly, Braje et al.'s results suggest that face recognition processes may be sensitive to variability in illumination direction. Recognizing a face is slower if that face is lit differently than it was when the face was recognized previously. Braje et al.'s results thus indicate that face recognition mechanisms may not be able to ignore variability in lighting, a result that could mean that face recognition processes rely on image-based representations.

Although each of these papers addresses a slightly different issue in object representation, I hope that the gestalt gives readers a sense of the current issues and results in the field. I also hope that this special issue will increase interest in both object recognition and the use of interdisciplinary approaches to study complex problems such as object recognition. 